\title{
The cognitive question in linguistics: An introduction to the distributed lan- guage approach
}

\author{
SUNE VORK STEFFENSEN
}

This article introduces the Distributed Language Approach (DLA), which is a contemporary framework in the language sciences. Building on recent developments in cognitive science, DLA takes a naturalistic perspective. According to this perspective, DLA rejects the view that the idea of 'language' correlates with a singular, ontological phenomenon. Instead, DLA takes its starting point in human ecology, including the behavioural coordination that takes place in human interactivity. This coordination is seen as radically heterogeneous, i.e. it is conditioned by multiple factors on different timescales (including embodied micro-dynamics and trans-situational resources). Further, DLA sees such coordinative actions as ecologically embedded, i.e. interwoven with the numerous activities that constitute human existence. The article opens with a review of how linguistics and the language sciences have incorporated insights from cognitive science in the second half of the $20^{\text {th }}$ century. It then discusses the recent developments in cognitive science that DLA draws on, in particular Distributed Cognition and Ecological Psychology. Finally, the article presents DLA by discussing five foundational assumptions in the framework. 